\title{
Successful Management of Paroxysmal Sympathetic Hyperactivity in Anti-N- Methyl-D-Aspartate Receptor Encephalitis
}

\author{
Chen Jing, Chiong Yi \\ Department of Rehabilitation Medicine, Singapore General Hospital, Academia level 4, 20 College Road, Singapore, 169856.
}

\author{
Corresponding Author: \\ Dr. Chen Jing \\ Email: chenjingsingapore@gmail.com
}

This is an Open Access article distributed under the terms of the Creative Commons Attribution License (creativecommons.org/ licenses/by/3.0).

Received

Accepted

Published

May 25, 2019

October 18, 2019

November 15, 2019

\begin{abstract}
Background: Paroxysmal sympathetic hyperactivity (PSH) is a hyper-adrenergic clinical syndrome that is predominantly described in acquired brain injuries, less commonly in other acquired brain injuries. The association of anti-N-Methyl-D-Aspartate Receptor (NMDAR) encephalitis and PSH is not well established. Case Report: We reported a PSH associated with anti-NMDAR encephalitis and the successful management of this case. A 32-year-old female presented with episodic involuntary movement and rapidly progressed to seizure and acute confusion. Further investigations confirmed the diagnosis of ovarian teratoma associated anti-NMDAR encephalitis which was treated with pulsed methyl-prednisolone, immunotherapy, and ovarian cystectomy. She developed episodic fever, tachycardia, hypertension, diaphoresis, and dystonia during her hospital stay. PSH was suspected after other causes were excluded and the diagnosis was made more probable by applying PSH assessment measure (PSH-AM). A combination of pharmacological agents was started with good response. At 9 months from symptoms onset, she achieved almost complete recovery and returned to work. Conclusion: This case report raises the awareness of PSH in anti-NMDAR encephalitis and illustrates the use of PSH-AM as an assessment tool to diagnose PSH and assess its severity. When PSH was recognized early and treated aggressively, good outcome can be achieved.
\end{abstract}

Keywords: Anti-N-Methyl-D-Aspartate Receptor Encephalitis, Dystonia, Hypertension, Seizures, Ovarian Teratoma.

\section{Introduction}

Paroxysmal sympathetic hyperactivity (PSH) is a distinct syndrome which is characterized by paroxysmal transient hyperthermia, tachycardia, hypertension, tachypnea, excessive diaphoresis and specific posturing [1]. PSH is commonly described in traumatic brain injury (TBI) but not commonly recognized in anti-NMDAR encephalitis and the reports have been sparse. This may be contributed by the absence of a universally accepted terminology as well as standard diagnostic criteria [2]. We report a case of PSH in a patient with antiNMDAR encephalitis and discuss the application of the recently developed assessment tool of PSH in the management of this case.

\section{Case Report}

A 32-year-old Chinese female presented to the Department of Neurology in a tertiary hospital with one day history of episodic involuntary choreiform movements and headache. The symptoms were preceded by an upper respiratory tract infection. Subsequently she developed acute confusion, agitation, and seizure. She did not have any medical or psychiatric illness, and was not on any medication or illicit drug prior to presentation. Extensive investigations were carried out including inflammatory and autoimmune markers, magnetic resonance imaging (MRI) of the brain, CT thorax, abdomen and pelvis, electroencephalography (EEG) and lumbar puncture. There was no evidence 
of infection of the central nervous system (CNS) and EEG did not show epileptic form activity. MRI brain was unremarkable. However, the antiNMDA receptor in both cerebral spinal fluid (CSF) and serum were positive. CT scan revealed a right ovarian tumor for which cystectomy was done and mature ovarian teratoma was confirmed histologically. The diagnosis of ovarian teratoma associated anti-NMDAR encephalitis was made. On day 5 of admission, the patient was started on first line treatment including intravenous immunoglobulins $21 \mathrm{gm}$ and methyl-prednisolone $1 \mathrm{~g} /$ day for 5 days followed by oral prednisolone in tapering dose. As her confusion and agitation still persisted 2 weeks after completion of treatment, she was considered as poor response to the first line treatment and was started on second line treatment including 3 cycles of IV cyclophosphamide 1000 $\mathrm{mg}$, and 6 cycles of rituximab $500 \mathrm{mg}$ from week 3 to week 10 .

Two weeks after symptoms onset she developed fever, sinus tachycardia, hypertension, diaphoresis, and episodic motor posturing without obvious precipitating factors. There were 5 to 6 episodes per day with each episode lasting 15 to 20 minutes. Her highest temperature was 38.7 degree celsius, heart rate was more than 140 beats per minute, respiratory rate was more than 30 breaths per minute, systolic blood pressure was more than $180 \mathrm{mmHg}$ [Fig.1]. Septic workup was not suggestive of an infective etiology. Her symptoms also did not resolve with empirical broad coverage antibiotics. PSH was suspected and PSH assessment measure (PSH-AM) [3] was applied to assess the likelihood of diagnosis. Her PSH-AM score was 26 which made the diagnosis of PSH probable [Fig.2]. The patient was started on oral clonazepam $0.5 \mathrm{mg}$ every night $(\mathrm{ON})$ and the dose was titrated to 1 mg three times per day (TDS) subsequently. After 1 week of clonazepam (on day 35 of admission), dystonia and diaphoresis improved significantly but fever, hypertension and tachycardia were still present episodically. PSH-AM score reduced

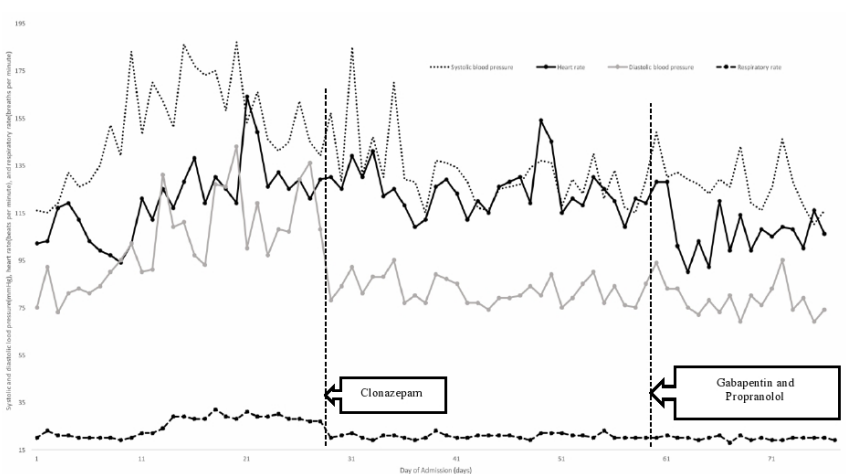

Fig.1: Patient's respiratory rate (RR), systolic blood pressure $(S B P)$, diastolic blood pressure $(D B P)$, and heart rate $(H R)$ after admission. Data point represents the daily highest record of patient's RR, SBP, DBP and HR after admission. After 2 weeks of admission, there was an increasing trend of RR, BP and HR. With starting clonazepam, there was a significant improvement in patient's $R R, D B P$. The $R R$ and $D B P$ decreased and stabilized, however SBP and HR were still on and off high. After starting gabapentin and propranolol, patient's SBP and HR normalized.

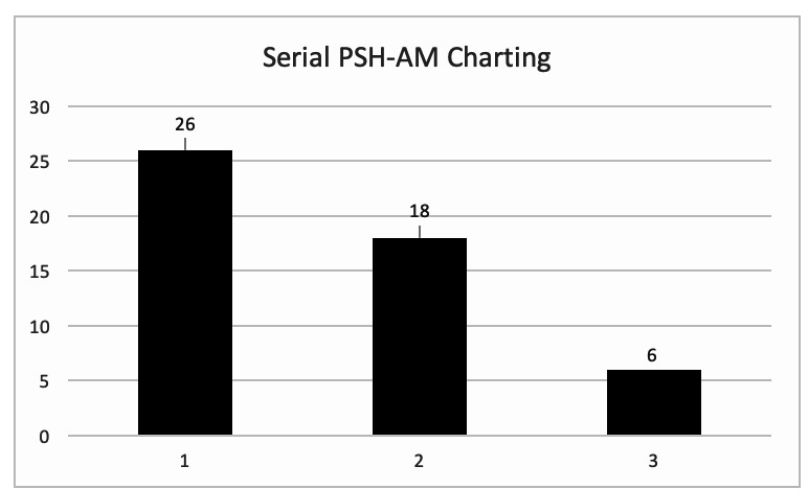

The figure showed that in our case, with pharmacotherapy PSH-AM score reduced.

Fig.2: Serial paroxysmal sympathetic hyperactivityassessment measure (PSH-AM) monitor in this case.

from 26 to 18 suggesting PSH was still probable [Fig.2]. Propranolol $20 \mathrm{mg}$ TDS, gabapentin 300 mg TDS were started and her symptoms resolved 1 week later. The PSH-AM assessment was repeated and the score decreased to 6 [Fig.2]. Clonazepam and gabapentin were gradually tapered down and stopped completely before discharge. Propranolol was continued at $20 \mathrm{mg}$ TDS upon discharge and stopped 3 months later. There was no obvious side effect observed from the medications. 
At 3 months from symptoms onset, her neurological and psychiatric symptoms improved significantly and she was discharged well. At 9 months from symptoms onset, she achieved almost complete recovery except subtle difficulties in auditory memory. She was independent in her activities of daily living and she returned to work.

\section{Discussion}

We reported $\mathrm{PSH}$ in a case with anti-NMDAR encephalitis, which is a relative rare condition in the literature. Anti-NMDAR encephalitis is one of the autoimmune encephalitis syndromes which present with prodromal headache and fever, followed by a multistage progression of symptoms that include seizures, decreased level of consciousness and memory deficits. The exact incidence of antiNMDAR encephalitis is unknown. In a study there were more than 400 cases reported in a threeyear period [4]. The diagnosis of anti-NMDAR encephalitis is confirmed by the detection of IgG antibodies to the GluN1 (also known as NR1) subunit of the NMDAR in serum or CSF [5]. It has a high association with ovarian teratoma presenting as paraneoplastic syndrome [6]. Treatment for antiNMDAR encephalitis includes tumor removal and immunotherapy. Good outcome can be expected with appropriate treatment of anti-NMDAR encephalitis. A retrospective study of 577 patients showed approximately 80 percent of patients achieved a good outcome by 24 months [6]. In our case, this patient had a typical presentation of antiNMDAR encephalitis for which she received tumor removal, steroids and IVIG as first-line therapy. She also received second-line therapy including rituximab and cyclophosphamide. Good clinical outcome was achieved with almost complete recovery at 9 months. Recognizing and successful managing PSH has contributed to the good clinical outcome of this case.

The association of PSH with anti-NMDAR encephalitis is not well established. A review of 349 PSH case reports found that about $80 \%$ of
PSH followed TBI, 10\% followed anoxic brain injury, 5\% followed stroke, and the remaining $5 \%$ occurred in association with hydrocephalus, tumors, hypoglycemia, infections, or unspecified causes [7]. To date there were only few case reports available describing the association of anti-NMDAR encephalitis with PSH $[8,9]$. Of note, although the cases formally diagnosed PSH was rare in antiNMDAR encephalitis, autonomic instability has been reported in anti-NMDAR encephalitis with a frequency of $69-89 \%$ in various case series $[10,11]$. The most common manifestations of autonomic instability reported are hyperthermia, cardiac dysrhythmias (tachycardia or bradycardia), hypersalivation, hypotension, hypertension, urinary incontinence, and sexual dysfunction [10]. We believe that the autonomic instability reported in anti-NMDAR encephalitis may often be PSH and $\mathrm{PSH}$ can be under-diagnosed and under reported in anti-NMDAR encephalitis.

There was no clear definition or terminology to describe PSH until 2014 when an international consensus was reached with a proposal of a clinical scoring system, namely, PSH-AM [3]. The PSHAM score is calculated by combining the clinical feature scale (CFS) score and diagnosis likelihood tool (DLT) score, which gives an estimate of the probability of a diagnosis of PSH [3]. In a case series [1], among 394 survivors of 521 patients admitted with acquired brain injury, 6 patients (1.5\%) were diagnosed as PSH by using PSH-AM. This study showed that PSH-AM provides a more objective measure to increase the clinical certainty of diagnosing PSH. The diagnosis of PSH in our case was supported by PSH-AM, and patient's progress was monitored by serial charting of PSHAM as well. From our experience, serial collection of PSH-AM score allows clinicians to assess the severity, monitor the progress of the patient's clinical status and response to treatment, therefore improve the efficacy in PSH management.

PSH can lead to serious complication if left untreated [2]. A wide range of pharmacological 
treatment has been used to manage symptoms of PSH such as opioids, benzodiazepines, betaadrenergic blockers, clonidine and gabapentin [2]. In practice, a combination of drugs is often needed to target the different components of PSH. The pharmacological agents chosen are largely dependent on the availability of the drugs and preference of the managing physicians as there is paucity of evidence available. In this case study, we found that propranolol, gabapentin and clonazepam were effective in controlling the symptoms of our patient. We also found that PSH-AM is a helpful measure as demonstrated in this case study as it allows us to diagnose PSH more confidently, guide the treatment and monitor the effects.

\section{Conclusion}

The diagnosis of PSH can be challenging in nontraumatic brain injuries and hence, often underrecognized. This is a case report of anti-NMDAR encephalitis complicated by PSH. This case report supported the use of PSH-AM for the diagnosis of $\mathrm{PSH}$ and to monitor its treatment effect. However as a single case report, it is difficult to assess the validity of PSH-AM. Larger studies are required to provide more evidence to support the use of PSH$\mathrm{AM}$ and to assess whether management guided by the measure can improve outcome of the patients. Furthermore, clinical experience and judgement are important to exclude other alternative diagnoses which can mimic PSH.

Contributors: CJ: manuscript writing, and patient management; $\mathrm{CY}$ : reviewing the literature and patient management. CJ will act as a study guarantor. Both authors have equal contribution to this case report and approved the final version of this manuscript.

Funding: None; Competing interests: None stated.

\section{References}

1. Godo S, Irino S, Nakagawa A, Kawazoe Y, Fujita M, Kudo D, et al. Diagnosis and management of patients with paroxysmal sympathetic hyperactivity following acute brain injuries using a consensus-based diagnostic tool: A single institutional case series. The Tohoku Journal of Experimental Medicine. 2017;243:11-18.

2. Meyfroidt G, Baguley IJ, Menon DK. Paroxysmal sympathetic hyperactivity: the storm after acute brain injury. The Lancet Neurology. 2017;16:721-729.

3. Baguley IJ, Perkes IE, Fernandez-Ortega JF, Rabinstein AA, Dolce G, Hendricks HT, et al. Paroxysmal sympathetic hyperactivity after acquired brain injury: consensus on conceptual definition, nomenclature, and diagnostic criteria. Journal of Neurotrauma. 2014;31:1515-1520.

4. Dalmau J, Lancaster E, Martinez-Hernandez E, Rosenfeld MR, Balice-Gordon R. Clinical experience and laboratory investigations in patients with anti-NMDAR encephalitis. The Lancet Neurology. 2011;10:63-74.

5. Pruss H, Dalmau J, Harms L, Höltje M, Ahnert-Hilger $\mathrm{G}$, Borowski $\mathrm{K}$, et al. Retrospective analysis of NMDA receptor antibodies in encephalitis of unknown origin. Neurology. 2010;75:1735-1739.

6. Titulaer MJ, McCracken L, Gabilondo I, Armangué $\mathrm{T}$, Glaser $\mathrm{C}$, Iizuka $\mathrm{T}$, et al. Treatment and prognostic factors for long-term outcome in patients with antiNMDA receptor encephalitis: an observational cohort study. The Lancet Neurology. 2013;12:157-165.

7. Perkes I, Baguley IJ, Nott MT, Menon DK. A review of paroxysmal sympathetic hyperactivity after acquired brain injury. Annals of Neurology. 2010;68:126-135

8. Hinson HE, Takahashi C, Altowaijri G, Baguley IJ, Bourdette D. Anti-NMDA receptor encephalitis with paroxysmal sympathetic hyperactivity: an underrecognized association? Clinical Autonomic Research. 2013;23:109-111.

9. Liu H, Jian M, Liang F, Yue H, Han R. Anti-N-methylD-aspartate receptor encephalitis associated with an ovarian teratoma: two cases report and anesthesia considerations. BMC Anesthesiology. 2015;15:150.

10. Dalmau J, Gleichman AJ, Hughes EG, Rossi JE, Peng $\mathrm{X}$, Lai M, et al. Anti-NMDA-receptor encephalitis: case series and analysis of the effects of antibodies. The Lancet Neurology. 2008;7:1091-1098.

11. Gable MS, Gavali S, Radner A, Tilley DH, Lee B, Dyner $\mathrm{L}$, et al. Anti-NMDA receptor encephalitis: report of ten cases and comparison with viral encephalitis. European Journal of Clinical Microbiology \& Infectious Diseases. 2009;28:1421-1429. 